\title{
CISIT le Campus Interdisciplinaire sur les Transports et la Mobilité Durable : focus sur les nouveaux défis pour des véhicules plus légers, fiables et sécuritaires
}

\section{CISIT the Interdisciplinary Cluster on Sustainable Transports and Mobility: Focus on new challenges for clean, safe and lightweight vehicles}

\author{
Eric Markiewicz \\ LAMIH, UMR CNRS 8201, Université de Valenciennes et du Hainaut Cambrésis, Le Mont Houy, \\ 59313 Valenciennes Cedex 9, Coordinateur du CISIT, http://www.cisit.org/
}

\begin{abstract}
This contribution presents CISIT, the cluster for sustainable mobility of people and goods. This successful initiative was set up in 2007 within the framework of the State-Region projects of contract and the FEDER Operational Program (CPERPO 2007-2013) for the French Nord-Pas de Calais region. CISIT unites the multidisciplinary scientific and technological skills of 12 regional research Labs and 3 technological development centres (about 360 researchers and engineers). CISIT's strategy consists, through industrial partnerships and in synergy with his ecosystem, in the long term development of scientific knowledge and innovative technologies (TRL 1-4 and 5) that can meet the future social needs in terms of mobility, safety, energy savings and environmental impacts. Here a focus is made on the results provides by one of the nine current actions: "new challenges for clean, safe and lightweight vehicles".
\end{abstract}

Résumé. Cette contribution présente CISIT, le cluster de la mobilité durable des personnes et des biens. Cette initiative réussie a été mise en place en 2007 dans le cadre des contrats de projets État-Région et du programme opérationnel FEDER (CPER-PO 2007-2013) pour la région Nord-Pas-de-Calais. CISIT unit les compétences scientifiques et technologiques pluridisciplinaires de 12 laboratoires régionaux de recherche et de 3 centres de développement technologique (environ 360 chercheurs et ingénieurs). La stratégie du CISIT consiste, au travers de partenariats industriels et en synergie avec son écosystème, dans le développement à long terme de connaissances scientifiques et de technologies innovantes (TRL 1-4 et 5) à même de répondre aux futurs besoins sociétaux sur la mobilité, la sécurité, les économies d'énergie et les impacts environnementaux. Ici, un accent est mis sur les résultats obtenus par l'une des neuf actions en cours: «nouveaux défis pour les véhicules propres, sûrs et légers ».
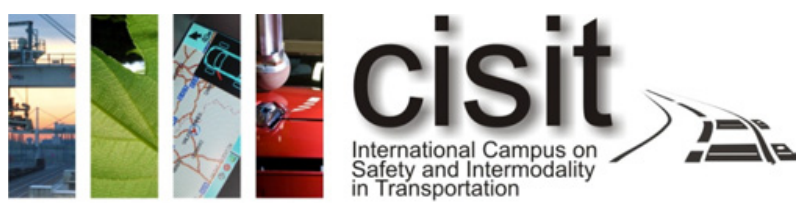
Safety and Intermodality in Transportation

\section{CISIT en quelques mots}

Afin de répondre aux attentes socio-économiques dans le domaine des transports, notamment en matière d'intermodalité, de sécurité, d'économies d'énergie et d'impact environnemental, cisit, le Campus International sur la Sécurité et l'Intermodalité dans les Transports, a été créé dans le cadre du Contrat de Projet Etat-Région 20072013.

Unique sur le territoire national et porteur d'actions de recherche et d'innovation à fort impact socio-économique, cisit fédère des compétences scientifiques et techniques interdisciplinaires de tout premier plan développées depuis 1983 au sein du Groupement Régional pour la Recherche dans les Transports (Fig. 1). La situation géographique de cisit, au cœur d'une région où la production ferroviaire et automobile d'une part, l'activité logistique d'autre part sont très importantes, en fait un partenaire idéal pour accompagner les industriels et grands opérateurs dans leurs développements stratégiques sur la mobilité durable des personnes et des biens.

Dans sa démarche de planification stratégique destinée à construire sa feuille de route, en cohérence et complémentarité avec celles des pôles de compétitivités et des grands projets nationaux et européens dédiés au transport, cisit met en avant les concepts de « sécurité »et de « co-modalité ». Ce dernier traduit l'usage efficace des différents modes de transport, tant de passagers que de fret, de façon à répondre à la fois aux besoins élevés de mobilité et au plus haut niveau d'exigence environnementale.

S'inscrivant pleinement dans les ambitions de la politique européenne des transports, cisit est un centre de référence pour innover les véhicules et les systèmes de transport de demain. Intelligents, communicants, fiables, sécuritaires et respectueux de l'environnement, tels sont les adjectifs qualifiants au mieux le transport tel que le conçoit cisit. Son action se décline en thèmes de recherche orientés vers une réponse aux trois grands enjeux sociétaux (Fig. 2). La logique de projets interdisciplinaires qui anime cisit a permis de prendre en compte leurs interactions inévitables : garantir la qualité globale de fonctionnement 
A multidisciplinary project which involves 4 main scientific fields

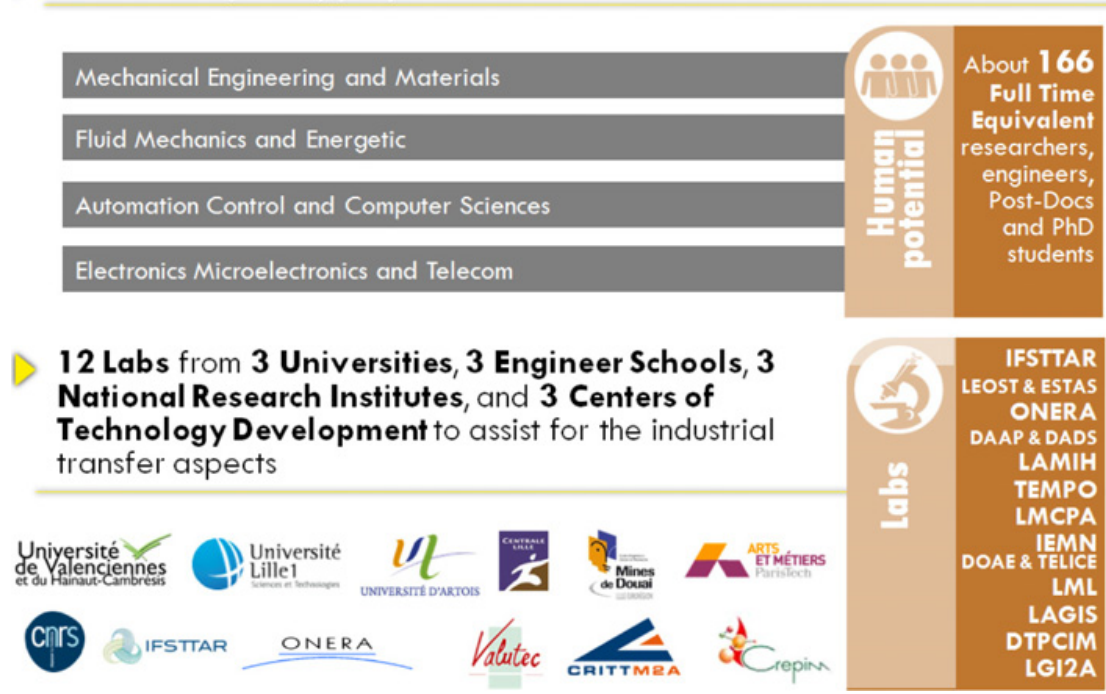

Figure 1. La communauté scientifique et technique du cisit.

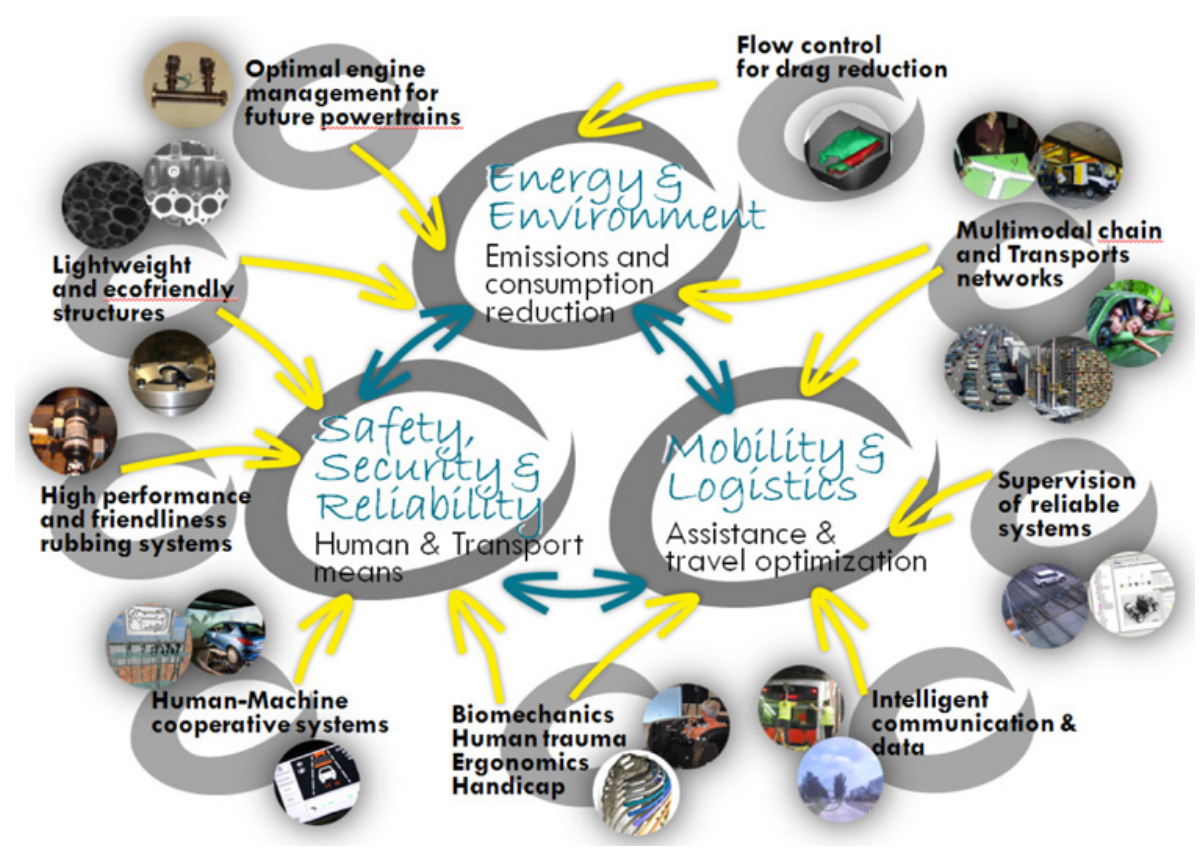

Figure 2. Impacts du cisit sur les trois grands enjeux sociétaux.

des systèmes par la prise en compte de la sécurité, de la sûreté et de la fiabilité; contribuer à une limitation des impacts environnementaux et de la consommation d'énergie sans affecter la qualité globale ; optimiser les systèmes de mobilité et de logistique.

Focus sur les nouveaux défis pour des véhicules plus légers, fiables et sécuritaires

Cette action traite de l'un des principaux enjeux de l'allégement des structures, à savoir le recours à des matériaux moins denses, mais sans dégradation de la performance fonctionnelle. Elle allie des expérimentations originales aux différentes échelles et des développements numériques afin d'améliorer les connaissances fondamen- tales nécessaires à l'utilisation de ces matériaux. Elle contribue ainsi à la réduction des émissions de gaz des véhicules par l'utilisation de matériaux plus légers tout en assurant un niveau de fiabilité et de sécurité optimal. L'établissement de la feuille de route stratégique de cette action, en concertation avec les partenaires industriels, a permis d'identifier les défis technologiques et scientifiques à relever.

\section{Défis technologiques}

> Durabilité et performance des nouveaux matériaux ( $\mathrm{Mg}, \mathrm{Al}, \mathrm{HSS}$, polymères, composites et matériaux sandwiches) sous sollicitations et environnements sévères (température, pression, vitesse). 

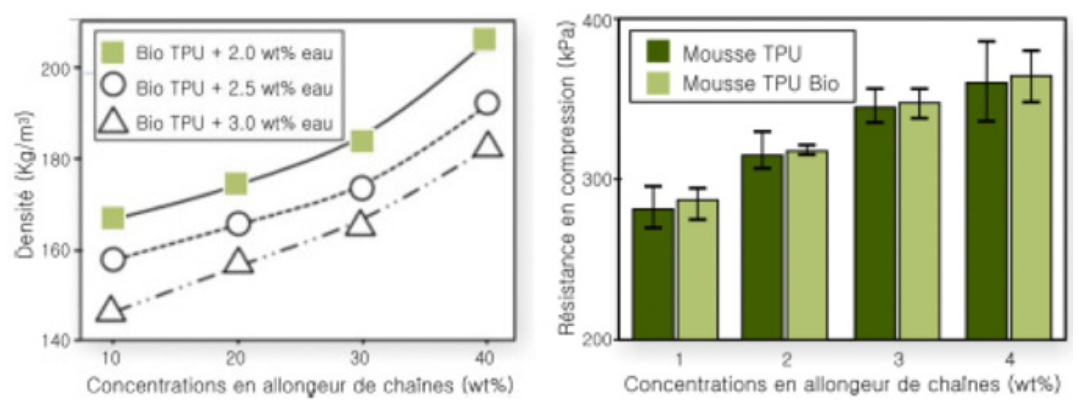

Figure 3. Effet des concentrations en allongeur de chaînes et en agent d'expansion sur la masse volumique des mousses polyuréthanes bio-sourcées. Comparaison des propriétés mécaniques en compression de mousses de polyuréthanes bio et non bio-sourcés.

> Hétérogénéités de comportement dues aux procédés de fabrication.

> Downsizing : étude de la performance des MPG.

> Résistance mécanique des assemblages et multiassemblages structuraux.

> Contrôle de santé structurale.

\section{Défis scientifiques}

> Nouvelles méthodologies et procédures d'essais appliquées aux nouveaux matériaux et aux systèmes d'assemblages. Cela comprend : le couplage matériau/ procédé, le couplage multi-physique et multi-échelle pour analyser le comportement quasi-statique et dynamique des matériaux sur de larges gammes de déformation et de vitesse de déformation plastique jusqu'à rupture, le comportement des matériaux en surface, le comportement sous chargement cyclique, ainsi que la prise en compte des hétérogénéités géométriques et matérielles.

> La modélisation du comportement et de la rupture de ces nouveaux matériaux et de la tenue des systèmes d'assemblage. Cela conduit à l'implémentation de nouveaux modèles aux différentes échelles pertinentes dans les simulations numériques.

\section{Quelques résultats marquants sur 2011-2012}

Génération de structures biosourcées à gradient par un procédé de rotomoulage/rotomoussage. Les formulations polyuréthanes massives, bio-sourcées ou non, développées précédemment ont été caractérisées. Dans tous les cas, la formation d'un polymère thermoplastique (impératif de recyclabilité) a été confirmée. Le rapport segments souples/segments rigides et les taux de catalyseurs ont été optimisés compte tenu de l'objectif en termes de temps et de température de réaction. De plus, des mousses de polyuréthane thermoplastique bio-sourcés expansées à l'eau ont été préparées en utilisant des allongeurs de chaînes bio-sourcés. L'effet de la formulation sur les propriétés physiques et morphologiques des mousses a été analysé (Fig. 3). Il a été montré que les propriétés mécaniques des mousses biosourcées fabriquées sont au moins équivalentes à celles des mousses élaborées à partir de précurseur issus de ressources fossiles.

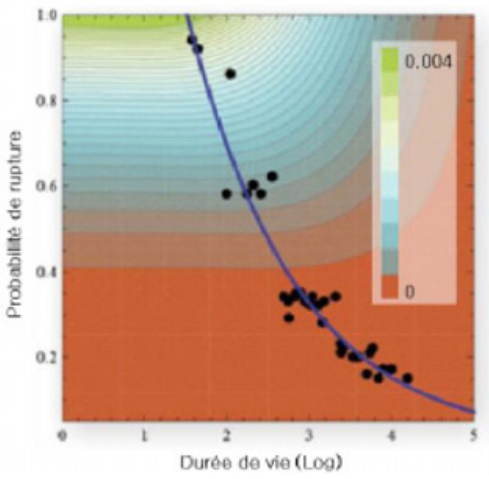

Figure 4. Critère probabilisé de fatigue thermomécanique.

Optimisation des procédés de fonderie pour l'allègement des culasses. L'endommagement et la durée de vie en fatigue thermomécanique des alliages d'aluminium obtenus par le Procédé de fonderie à Modèle Perdu (PMP) ont été étudiés. Ces alliages sont utilisés dans l'industrie automobile entre autres en vue de réduire la masse des culasses. Les propriétés mécaniques et la durée de vie en fatigue de ces matériaux sont largement influencées par la microstructure. Plusieurs critères de fatigue macroscopiques ainsi qu'un scénario de mécanisme d'endommagement à l'échelle microstructurale ont été proposés et l'approche initialement déterministe a été étendue à la détermination de probabilités de rupture à partir d'une étude statistique de la microstructure et d'une loi de propagation de fissure similaire à une loi de Paris (Fig. 4). Les méthodes et les critères proposés sont alors en bon accord avec les résultats expérimentaux sur des éprouvettes et des structures industrielles élémentaires.

Nouveaux outils pour la détection de l'endommagement dans les matériaux métalliques. De nombreuses études ont été menées sur les mécanismes de déformation et d'endommagement à l'échelle de la microstructure de matériaux métalliques. Elles se fondent en grande partie sur des mesures de champs cinématiques et thermiques à hautes résolutions spatiales, thermiques et temporelles. Ainsi, des résultats originaux ont permis de montrer le rôle important joué par les interfaces (joints de grain, joints de macle) ainsi que par les désorientations cristallographiques dans les mécanismes de localisation de la déformation dans les aciers (Fig. 5). Des travaux ont également démarré dans le cas des alliages d'aluminium utilisés dans les composants moteur de l'automobile 


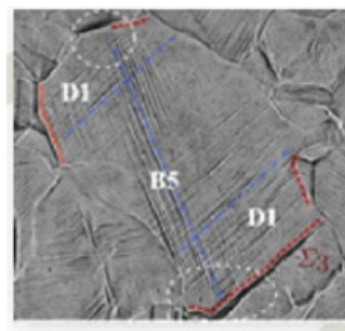

a Micrographie après essai

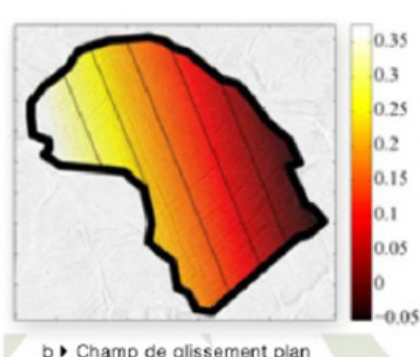

b. Champ de olissement plan

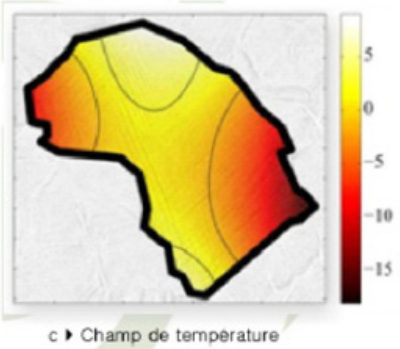

c Champ de temperature

Figure 5. Analyse granulaire des couplages thermomécaniques dans un polycristal métallique.
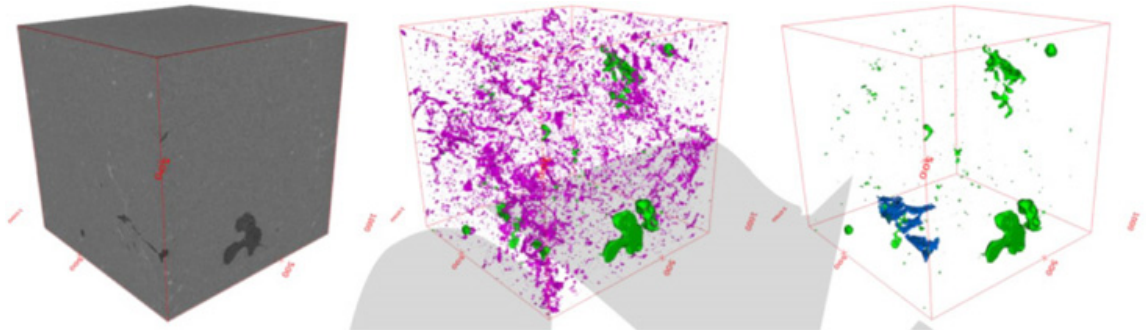

Figure 6. Reconstruction microtomographique d'un alliage $\mathrm{AlSiCu}$ avec au centre, les intermétalliques, à droite, les porosités.
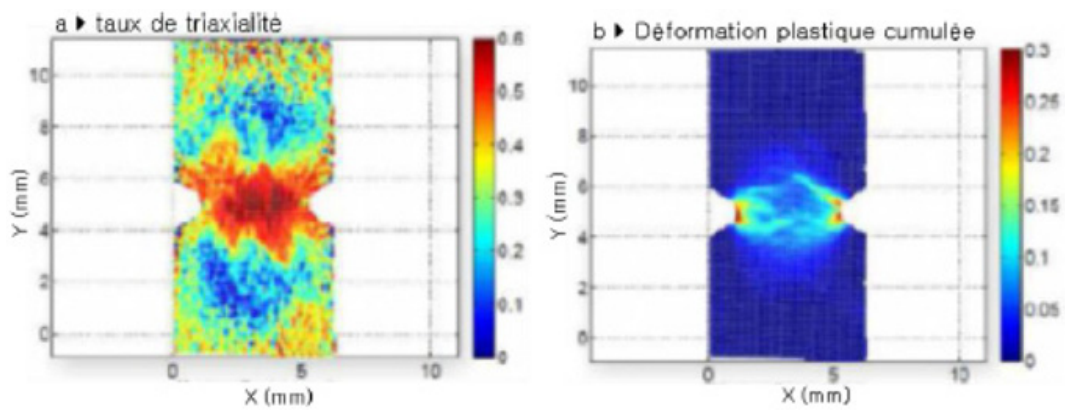

Figure 7. Cartographies du taux de triaxialité (a) et de la déformation plastique cumulée (b) sur éprouvette plate entaillée Ti6Al4V.

(i.e. culasse, Fig. 6) pour lesquels la microtomographie, permettant des mesures de champs 3D, va devenir un outil central d'investigation. Ils s'appuient sur les compétences acquises dans le cadre de cisit en métrologie optique microstructurale (équipements acquis et développements réalisés dans les phases précédentes de cisit) et surtout sur la future plateforme régionale de microtomographie RX ISIS4D, mutualisée entre les partenaires cisit de cette action (livraison en juin 2013). Cela permet de confirmer cisit comme un acteur majeur dans le domaine de la caractérisation 2D/3D des matériaux du transport en conditions expérimentales.

Caractérisation du comportement dynamique des alliages de titane par la Méthode des Champs Virtuels (MCV). Le comportement de matériaux subissant des chargements dynamiques à hautes énergies (crash, impacts, explosions) est généralement sensible à la vitesse de déformation. Les procédures classiques de caractérisation des modèles de comportement utilisent des essais statiquement déterminés nécessitant le respect d'hypothèses limitatrices et contraignantes. Ainsi, le traitement de champs mécaniques hétérogènes est impossible, ce qui limite l'exploitation des essais à de faibles gammes de déformations et oblige à réaliser plusieurs tests à vitesse de déformation constante pour la caractérisation de la viscoplasticité. Enfin, ces restrictions empêchent de profiter pleinement de la quantité importante d'informations accessible par la mesure de champs cinématiques appliquée à la dynamique rapide. Une solution est alors de recourir à des essais statiquement indéterminés, permettant d'exploiter des champs hétérogènes. Parmi les outils disponibles, la Méthode des Champs Virtuels (MCV) présente des avantages certains par rapport aux méthodes classiques de recalage par éléments finis. Les travaux ont porté sur le développement de la MCV pour la caractérisation du modèle viscoplastique de Johnson-Cook d'un alliage de titane Ti6Al4V. Un des grands atouts de la MCV est qu'elle permet de réduire à deux le nombre d'essais requis en exploitant l'hétérogénéité des champs de déformations et de vitesses de déformations d'une éprouvette plate entaillée. Le premier, en conditions de chargement QS, permet d'identifier les paramètres de plasticité indépendante du temps. Ensuite, un essai dynamique sur vérin hydraulique permet, grâce aux effets de localisation dus à la présence de l'entaille, d'identifier les paramètres viscoplastiques sur une large gamme de vitesses de déformation de [0, $300 \mathrm{~s}-1]$ (Fig. 7).

Caractérisation de lubrifiants respectueux de l'environnement. Les écoulements d'un lubrifiant respectueux de l'environnement entre la surface rugueuse 


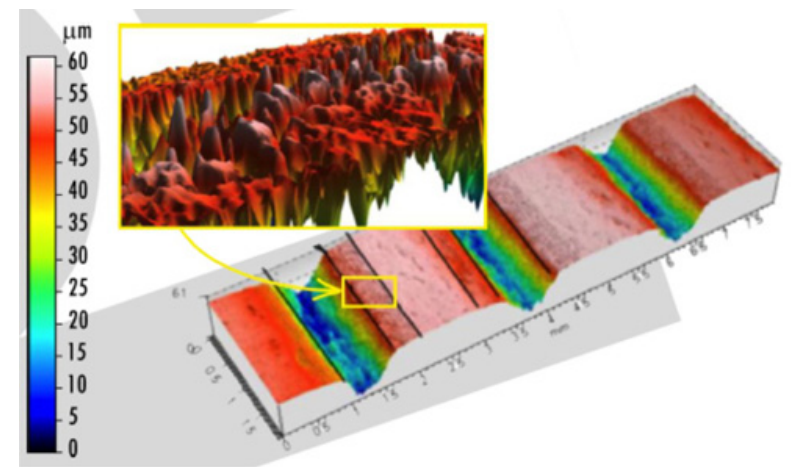

Figure 8. Signature du régime de lubrification en mise en forme de pièce en aluminium.

d'une barre en aluminium et un outil lisse en verre sont étudiés expérimentalement sur le banc d'étirage de barre développé par Bay et al. La rugosité des barres d'aluminium est mesurée par l'interféromètre Zygo NewView 7300 acquis dans le cadre du cisit. Lors de tels contacts, la rugosité de contact évolue, laissant apparaitre une signature caractéristique du procédé tribologique (Fig. 8). Le système expert MesRug a montré que les paramètres «classiques» de rugosité, tel que le Ra ou le Rt, sont incapables de détecter cette signature tribologique. Par contre, la courbe moyenne des pics de rugosité met clairement en évidence la variation des régimes de lubrification à l'échelle des aspérités.

Température critique pour la formation des couches d'oxydes en laminage à chaud. Les couches d'oxydes se forment sur les surfaces métalliques spécifiquement en laminage à chaud $\left(>1000^{\circ} \mathrm{C}\right)$. La qualité des produits peut être impactée par ces oxydes qui restent sur les pièces ou collent à l'outillage. Une méthodologie originale a été proposée pour caractériser les oxydes à haute température, pression et gradient de déformation, utilisant le simulateur thermomécanique Gleeble et le prototype MeBoOS développé en 2010 grâce aux financements cisit. Pour les aciers au Chrome, de nombreuses porosités existent à l'intérieur de la couche d'oxydes, avec des concentrations d'oxydes de fer, de chrome et de silicium à l'interface. Les résultats montrent l'existence d'une température critique pour laquelle les couches d'oxydes arrêtent de croitre (Fig. 9). Pour des températures supérieures à $1050^{\circ} \mathrm{C}$, les oxydes de chrome modifient le comportement de la couche d'oxydes et particulièrement son aptitude à la fissuration.

Nouvel élément cohésif pour la modélisation des assemblages collés sous chargement dynamique rapide. Les structures collées sont de plus en plus utilisées dans les industries automobiles et d'aviation. Cette technique a l'avantage d'être facile à utiliser, de permettre l'assemblage de matériaux de natures différentes et d'introduire une continuité mécanique dans le comportement. Néanmoins, en calcul des structures par Elément Finis $(\mathrm{EF})$ le recours à des macroéléments de liaison est indispensable afin de ne pas pénaliser les temps CPU. Un élément cohésif dédié pour les matériaux à comportements visqueux a été développé et validé par comparaison avec des résultats sur des

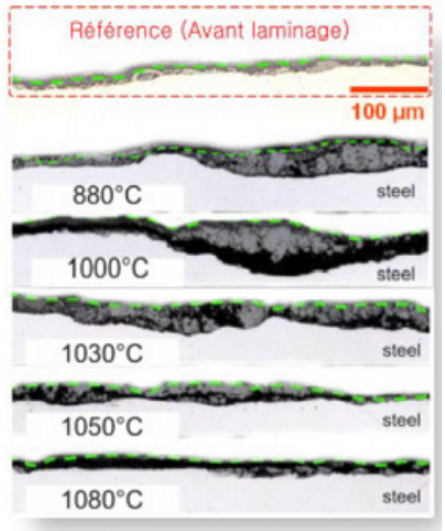

Figure 9. Evolution de l'épaisseur de la couche d'oxydes en fonction de la température de laminage.

crash-box. Cet élément a l'avantage de reproduire le comportement physique du matériau en tenant compte de son comportement visqueux, endommageable et sensible à la pression hydrostatique. D'autre part, un protocole expérimental est actuellement développé, utilisant les barres de Hopkinson en vue de réaliser des essais dynamiques locaux en mode I, II et mixte afin de déterminer la rupture du joint de colle et d'améliorer la prédiction de la vitesse de propagation de la fissure dans le joint de colle (Fig. 10).

Développement de polymères fonctionnalisés (piézoélectriques) et fabrication de capteurs par injection ou extrusion multi-composants. Le développement de capteurs ultrasonores conformables, c'est-à-dire aptes à s'adapter aux structures de formes complexes, présente un intérêt industriel majeur pour l'élaboration de structures « intelligentes », notamment dans le domaine des transports. Leur souplesse leur permettrait en effet de subir sans dommage, contrairement aux céramiques piézoélectriques classiques rigides (PZT), les dilatations des structures au cours de leurs exploitations (dilatation thermique, déformation élastique lors des sollicitations mécaniques, ....). La faisabilité de l'élaboration de ces capteurs à partir d'un polymère bio-sourcé intrinsèquement piézoélectrique (PA 11), fonctionnalisé par des charges piézoélectriques permettant d'en accroitre l'impédance acoustique, a été montrée. Ce projet s'appuie sur la plateforme de plasturgie et de caractérisation et la plateforme d'élaboration et de caractérisation de céramiques des partenaires cisit. En 2012, les travaux ont permis de clarifier la nature cristalline nécessaire pour des propriétés piézoélectriques optimales des films de PA 11 (phase smectique $\delta^{\prime}$ ) et le type de procédé de fabrication (extrusion cast suivi d'un étirage à très faible vitesse - Fig. 11).

\section{CISIT ET L'APRÈS 2013}

cisit a d'ore et déjà préparé sa feuille de route de l'après 2013 en collaboration avec l'ensemble de ses partenaires et avec le concours de spécialistes en gestion de projets. L'évolution de l'écosystème régional a mis en évidence le positionnement amont, en termes de recherche, du cisit et sa fonction d'irrigation de l'innovation par la recherche. 

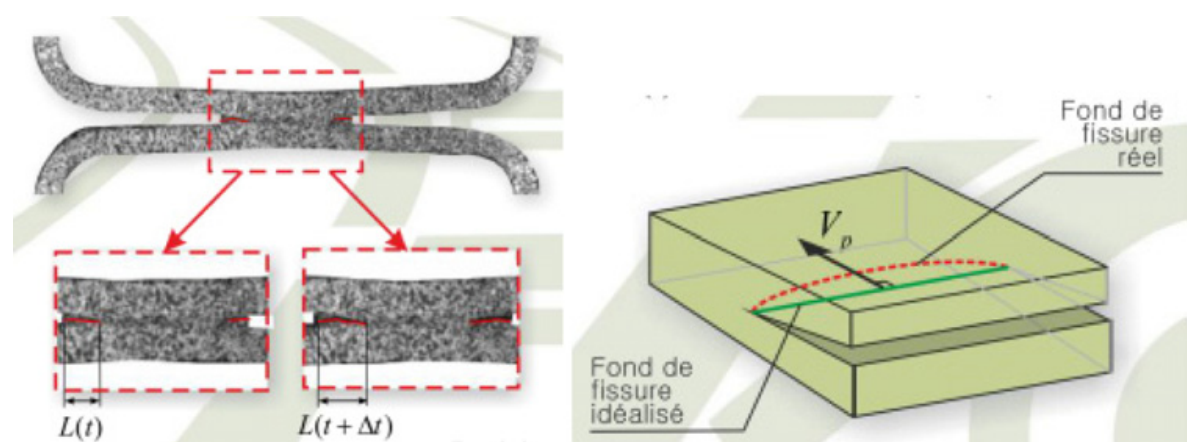

Figure 10. Mesure de la vitesse de propagation de la fissure en mode I.

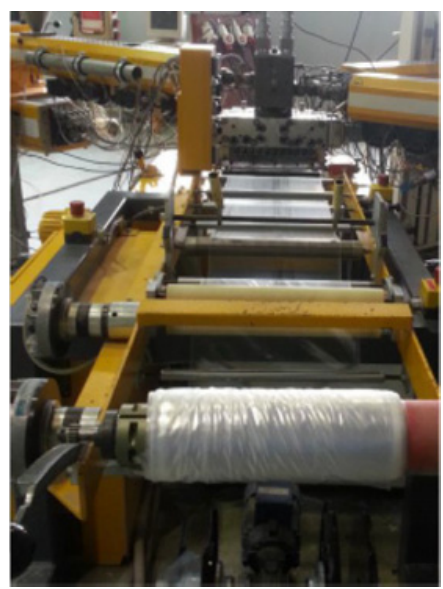

Figure 11. Mise en forme des films bio-sourcés piezoélectriques.

Pour 2014-2020, la vision et l'ambition partagées entre les partenaires est d'afficher, d'ici 2020, le cisit comme un acteur européen sur l'enjeu de la mobilité durable des personnes et des biens, reconnu pour l'originalité de son approche, l'excellence de sa recherche et son lien avec les industriels et les opérateurs de transport. La contribution à la définition d'offres de formation opérée conjointement par les établissements membres du cisit est également un objectif poursuivi afin d'assurer le continuum formationrecherche-innovation.

Pour atteindre cet objectif, la stratégie scientifique du cisit vise à renforcer son offre de recherche sur le moyen-long terme, notamment en accentuant son effort de développement de nouvelles connaissances et technologies génériques, à mêmes d'être mises à profit dans la chaine de l'innovation en synergie et en articulation complètes avec son écosystème régional L'offre 2014-2020 se concentrera sur quatre cibles stratégiques déclinées en thèmes de recherche (Fig. 12) orientés vers une réponse aux trois grands enjeux sociétaux. La poursuite d'une logique de projets interdisciplinaires qui anime cisit permettra de prendre en compte leurs interactions inévitables. Il est à noter une implication importante et fondamentale de la communauté scientifique des SHS, afin de prendre en compte à l'amont de tout projet d'innovation, l'ergonomie, la psychologie, les facteurs humains, les dimensions juridiques, économiques et le management de l'innovation.

Afin d'assurer une plus grande capacité opérationnelle d'animer sa communauté, de suivre ses projets, d'assurer le lien avec les financeurs institutionnels, les industriels

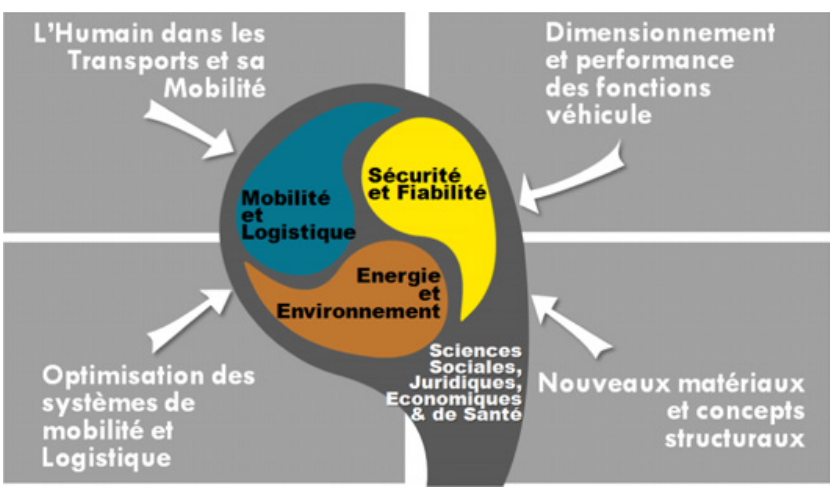

Figure 12. Cibles stratégiques vs. enjeux sociétaux 2014-2020 de cisit.

et les opérateurs de transport, de pouvoir communiquer en son nom pour accroître sa lisibilité et sa visibilité, de pouvoir se positionner clairement dans l'écosystème, cisit se dotera d'une structure avec une identité juridique. Un premier consensus entre les partenaires est celui d'une entité juridique sans personnalité morale portée par une structure souple et légère permettant : une collaboration entre les équipes (unités, ou équipes dans les unités); une capacité à la fois d'identifier un positionnement disciplinaire, d'établir des collaborations ; d'inciter à répondre aux appels à projets nationaux et internationaux (souvent transverses); de créer une véritable politique de recherche transversale entre les Sciences pour l'Ingénieur, les Sciences et Technologies de l'Information et de la Communication, les Sciences Humaines et Sociales, les Sciences Juridiques, les Sciences Économiques, les Sciences de Santé ; de pouvoir associer très largement les organismes de recherche nationaux (CNRS, IFSTTAR, ONERA, INRIA...). Une Fédération de Recherche, préférablement portée par le CNRS, semblent plus à même de conférer la souplesse désirée et une meilleure visibilité notamment vis-à-vis des organismes de recherche et des administrations de tutelle. Celle-ci est à l'étude . . .

\section{Références}

www.cisit.org (présentation et rapports d'activités) SRI du Nord-Pas de Calais, décembre 2009.

SNRI, 2009. 
Proposal for a Council Decision establishing the Specific Programme Implementing Horizon 2020 - the Framework programme for Research and Innovation (2014-2020), Bruxelles, 30.11.2011.

Roadmaps des plateformes européennes ERRAC, ERTRAC et ACARE.
Alliances ANCRE et ALLENVI, et programmes ANR liés.

Contribution française sur les priorités scientifiques et technologiques pour le cadre stratégiques commun de recherche et d'innovation «Horizon 2020», septembre 2011. 